\title{
EDITORIAL
}

\section{On pulmonary rehabilitation and the flight of the bumblebee: the ATS/ERS Statement on Pulmonary Rehabilitation}

\author{
L. Nici*, R. ZuWallack\# ${ }^{\#}$, E. Wouters ${ }^{\top}$ and C.F. Donner ${ }^{+}$
}

$\triangle$ ccording to conventional aerodynamic principles, the bumblebee cannot fly. Yet these fuzzy creatures indeed do fly effortlessly from plant to plant gathering pollen in our gardens. This paradox was resolved only when it became clear that the bumblebee's flight is more like a helicopter than an aeroplane. Using similar logic, pulmonary rehabilitation (PR) should not work since it has no direct effect on the forced expiratory volume in one second. Yet this comprehensive intervention demonstrably improves symptoms, exercise performance and quality of life, and decreases healthcare consumption in individuals with chronic respiratory disease. This apparent paradox was resolved when it became clear that patients with chronic respiratory disease experience substantial morbidity from secondary impairments, such as peripheral muscle, cardiac, nutritional and psychosocial dysfunction, which can be mitigated by PR.

In recent years, significant advances in the understanding of the primary and secondary impairments associated with chronic respiratory diseases, with particular focus on chronic obstructive pulmonary disease (COPD), have led to a better and increasing consideration of the role played by PR as an integral and important part of disease management. Since the 1997 European Respiratory Society (ERS) [1] and 1999 American Thoracic Society (ATS) [2] Statements on PR, there has been a dramatic increase in the science supporting the effectiveness of this therapeutic approach. Laboratory studies as well as randomised, controlled trials using valid and reproducible outcome measures have provided insight into the reasons why PR works. This rapidly growing evidence base and its implications for the care of patients with chronic respiratory disease led to the development of the current ATS/ ERS Statement on PR, published in the American Journal of Respiratory and Critical Care Medicine [3]. This international document presents recent scientific advances in our understanding of the multisystemic effects of chronic respiratory

\footnotetext{
*Providence Veterans Administration Medical Center and Brown University, Providence, Rl, and \#Section of Pulmonary and Critical Care Medicine, St. Francis Hospital \& Medical Center, Hartford, CT, USA. "Dept of Pulmonology, University Hospital, Maastricht, The Netherlands. ${ }^{+}$Mondo Medico, Multidisciplinary and Rehabilitation Outpatient Clinic, Borgomanero, Italy.
}

CORRESPONDENCE: C.F. Donner, Mondo Medico, Multidisciplinary and Rehabilitation Outpatient Clinic, Via Monsignor Cavigioli, 10, 28021 Borgomanero (NO), Italy. Fax: 39 0322869950. E-mail: cfdonner@mondomedico.it disease and outlines how PR addresses the resultant functional limitations.

The Statement provides a new definition of PR, as follows: "Pulmonary rehabilitation is an evidence-based, multidisciplinary, and comprehensive intervention for patients with chronic respiratory diseases who are symptomatic and often have decreased daily life activities. Integrated into the individualized treatment of the patient, pulmonary rehabilitation is designed to reduce symptoms, optimize functional status, increase participation, and reduce health care costs through stabilizing or reversing systemic manifestations of the disease" [3]. PR involves patient assessment, exercise training, education, nutritional intervention, psychosocial support and outcomes assessment. It includes a spectrum of intervention strategies directed at the primary and the secondary impairments associated with chronic respiratory disease. PR should be integrated into the lifelong management of the respiratory patient, providing the opportunity to coordinate care over the entire clinical course of the disease. Thus, components of PR (but not necessarily the programme as traditionally considered) should be prescribed, depending on the specific need. These may range from promoting smoking cessation in Global Initiative for Chronic Obstructive Lung Disease (GOLD) stage 0 COPD to providing palliative care in end-stage lung disease.

The Statement, endorsed by both the ATS and the ERS, is, for the first time, the result of collaborative efforts of a multidisciplinary group of PR healthcare professionals from Europe and the Americas, defining a common perspective in a field with sometimes different and even opposing views and modes of practice. Contributions came from both academics and clinicians. This diversity in authorship led to lively discussion, but ultimately strengthened the document through widening its applicability. As it stands, the Statement is positioned between, and complementary to, the 1997 American College of Chest Physicians and American Association of Cardiovascular and Pulmonary Rehabilitation (AACVPR) Evidence-Based Guidelines on Pulmonary Rehabilitation [4], and the 2004 AACVPR Guidelines for Pulmonary Rehabilitation Programs [5]. The former focuses on the available evidence base supporting PR and its components, whereas the latter emphasises how to optimise PR in a guidelines format.

The scientific evidence supporting each component of PR varies widely. For example, there is considerably more science 
from randomised, controlled trials for exercise training than for psychosocial support. This, however, does not imply that the latter is not effective; rather this accepted component has not yet been rigorously tested to the same degree. Reflecting this variability, the Statement contains both evidence-based science and expert opinion; as the science expands, new versions will shift toward the evidence-based end of the spectrum. Most clinical trials in PR have focused almost exclusively on patients with COPD. However, treatable comorbidities (such as peripheral muscle dysfunction) are common to all chronic respiratory disease, and collaborative self-management strategies can be broadly applied. Therefore, PR should be beneficial in a wide variety of diseases. The evidence for PR in these settings is slowly developing and, where available, is included in the Statement.

Exercise training, widely regarded as the cornerstone of PR, is the most potent intervention available to improve exercise tolerance in individuals with chronic respiratory disease. Scientific research has shed new light on the physiological underpinnings of exercise limitation, and the Statement discusses ventilatory, gas exchange, cardiac, skeletal muscle and respiratory muscle abnormalities. This is followed by approaches aimed at optimising the effectiveness of exercise training, including duration and frequency, intensity, specificity and modality (i.e. endurance, strength). The Statement highlights additional strategies that may be used in selected patients to enhance training effects, including maximising pulmonary function at the onset, supplemental oxygen (possibly even for nonhypoxaemic patients with COPD), noninvasive mechanical ventilation, respiratory muscle training and neuromuscular electrical stimulation.

Body composition abnormalities, especially depletion of fatfree mass, are prevalent in patients with advanced respiratory disease and significantly contribute to morbidity and mortality. Exercise training, with its higher caloric requirements, may further aggravate these abnormalities. Conversely, obesity is associated with increased work and oxygen cost of breathing. The Statement discusses the rationale for identifying, addressing and treating body composition abnormalities in order to enhance the benefits of PR.

Adherence to therapeutic modalities is a crucial health behaviour in the management of chronic respiratory disease, with the most effective interventions designed to improve selfmanagement capabilities. The Statement stresses the importance of this form of educational intervention in PR. Long-term continuation of increased levels of exercise and activity are of utmost importance in PR, and their promotion should be incorporated into a management plan early in the course of therapy. Self-management education should also include instruction in the prevention and early treatment of respiratory exacerbations, breathing strategies, coping techniques and end-of-life decision making. The goal of all these strategies is to effect a permanent alteration in lifestyle, integrating healthcare habits into the lifelong management of patients with chronic respiratory disease and structuring a dynamic collaboration between patient, family and healthcare providers.

In addition to providing scientific rationale and clinical expertise, the American Thoracic Society/European Respiratory Society Statement on Pulmonary Rehabilitation looks to the future. Given the current evidence, we no longer need to prove that pulmonary rehabilitation works; instead, we need to focus on ways to improve its effectiveness and broaden its applicability. Enhancing effectiveness will require a thorough understanding of the unique problems of the individual respiratory patient and new modalities to target these problems. Pulmonary rehabilitation should be made available to all who need it, and this will require education and advocacy in both healthcare and government institutions. New studies must address ways to maintain the benefits of pulmonary rehabilitation. This will require, at a minimum, a better understanding of human behaviour and ways to modify it. Perhaps we need to think of pulmonary rehabilitation as a long-term strategy rather than a short-term intervention. We look forward to these endeavours and others that will refine the process of pulmonary rehabilitation and expand its scope.

\section{REFERENCES}

1 Donner CF, Muir JF. Selection criteria and programmes for pulmonary rehabilitation in COPD patients. Rehabilitation and Chronic Care Scientific Group of the European Respiratory Society. Eur Respir J 1997; 10: 744-757.

2 American Thoracic Society, Pulmonary rehabilitation - 1999. Am J Respir Crit Care Med 1999; 159: 1666-1682.

3 Nici L, Donner C, Wouters E, et al. American Thoracic Society/European Respiratory Society Statement on Pulmonary Rehabilitation. Am J Respir Crit Care Med 2006; 173: 1390-1413.

4 ACCP/AACVPR Pulmonary Rehabilitation Guidelines Panel, American College of Chest Physicians. American Association of Cardiovascular and Pulmonary Rehabilitation. Pulmonary rehabilitation: joint ACCP/ AACVPR evidence-based guidelines. Chest 1997; 112: 1363-1396.

5 Sanderson BK, Southard D, Oldridge N. AACVPR consensus statement. Outcomes evaluation in cardiac rehabilitation/secondary prevention programes: improving patient care and programe effectiveness. J Cardiopulm Rehabil 2004; 24: $68-79$. 